\title{
Cómo se aprende: entre cognición, construcción y metacognición
}

Piergiuseppe Ellerani*

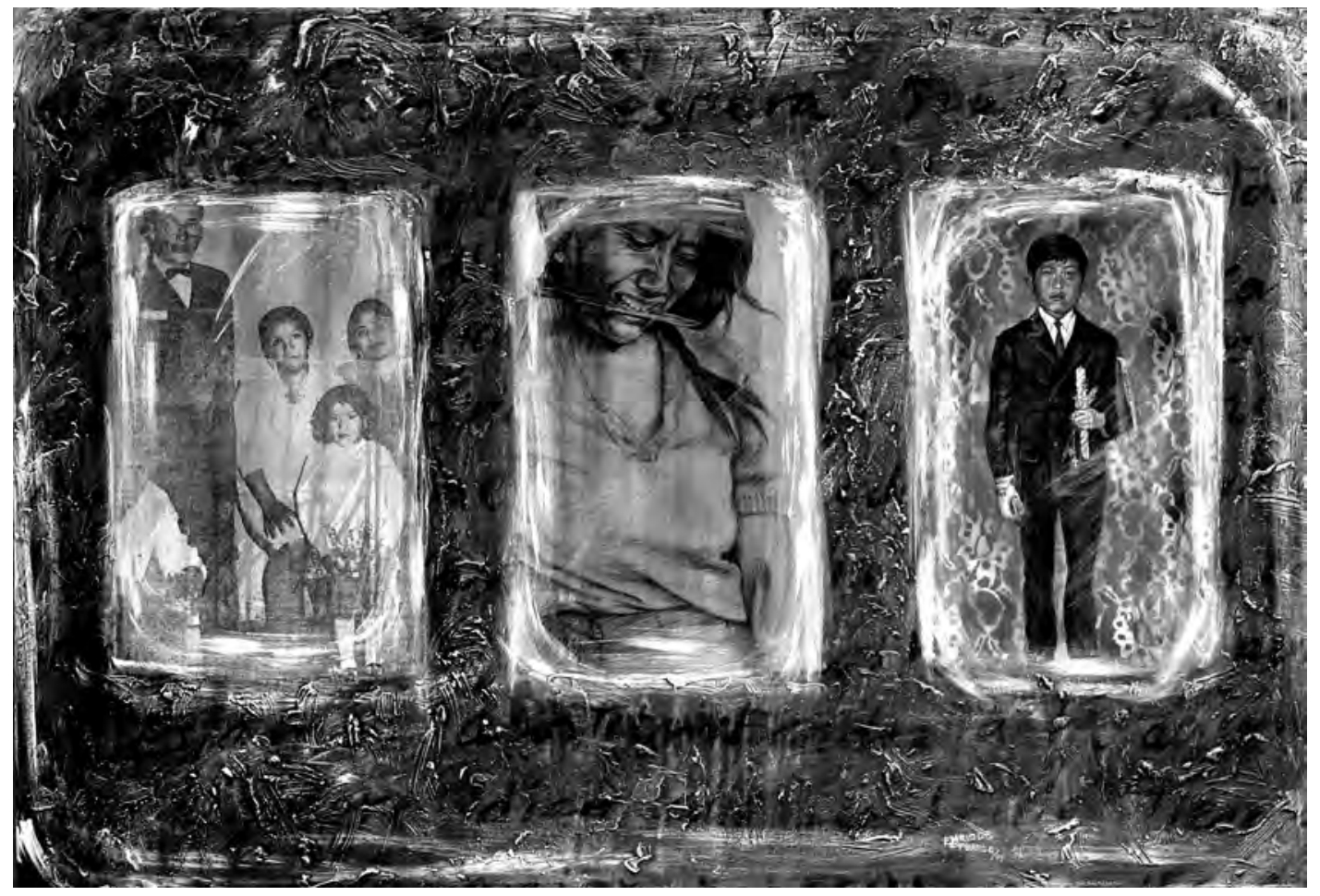

Memoria en. Acrílico sobre tela. 1998

\section{Introducción}

Los nuevos desafíos con los cuales el sistema de instrucción y formación tiene que enfrentarse como consecuencia del cambio y de la com- plejidad de la sociedad actual, ponen de manifiesto temáticas correlacionadas con: $a$. Los significados de la educación y de la formación moderna; $b$. El desarrollo del capital humano y social; $c$. El desarrollo de todas las inteligencias y de

* Doctor en Ciencias de la Educación. Investigador de la Universidad de Bolzano. Director científico del curso DiDUPS ofertado por Edulife. Coordinador del sitio educativo http://www.scintille.it. Forma parte del grupo italiano de investigación sobre el Cooperative Learning con sede all'ISRE di Venezia. 
hábitos mentales complejos; $d$. La construcción de unos contextos generativos del aprendizaje.

La escuela y la universidad pueden volverse un contexto intencional de educación para conseguir inteligencias plurales, aptas para pensar de manera autónoma, flexible y creativa, y para solucionar los cada vez nuevos problemas procedentes de la vida en la sociedad. En este contexto, es posible actuar con el fin de conseguir que los discentes dominen competencias cada vez más elevadas, a través de la definición de currículos para la vida. La organización de ambientes en los cuales el aprendizaje y la comprensión se logren como descubrimiento se inspira en un planteamiento pedagógico constructivo e investigador, que considera al discípulo como un alumno activo, social e individualmente diferente. En términos educativos se adopta el lema de que la inteligencia puede incrementarse y asimismo de que es posible formar al educando para que adquiera disposiciones mentales que determinen comportamientos adecuados en situaciones complejas.

Ha tomado un papel de gran envergadura la reflexión sobre "cómo se aprende y sobre qué se aprende", poniendo de manifiesto la contribución aportada por los estudios sobre la metacognición.

Formar a los discentes para la complejidad y educarlos para que adquieran actitudes metacognoscitivas correlacionadas cada vez más con procederes adelantados y competentes - para desarrollar a cada "ser humano", y que se vuelva así un capital inviolable, único e irreducible en la sociedad compleja- constituye un desafío al cual el sistema formativo tendría que reconocer primordial importancia. En efecto, hoy día muchos estudios que ponen de manifiesto que el éxito formativo, el crecimiento de la autoestimación y los resultados positivos en los aprendizajes están correlacionados con la actitud de colocar en el centro de la atención al educando que aprende con una competencia metacognoscitiva igualmente satisfactoria.

Sin embargo, esta postura estimulante nos plantea algunas preguntas: ¿Cómo pueden in- corporarse las investigaciones más adelantadas sobre la cognición humana, el desarrollo y el aprendizaje a las prácticas de la organización de las actividades educativas y formativas? ¿Cómo se puede envolver a los educandos en los procesos de aprendizaje y en la motivación, con el fin de acrecentar su éxito total en la escuela y en la universidad?

Son estas interrogantes las que vuelven apasionante el trabajo en la enseñanza, que se coloca en un contexto de profundas transformaciones sociales y culturales, como aquellas que han empezado a realizarse en el primer quinquenio del siglo XXI.

Hoy día se considera al aprendizaje como un proceso activo de construcción de conocimientos, habilidades y actitudes en un contexto de interacción del discípulo con los docentes, los compañeros y los medios didácticos. En el ámbito del mismo se da forma: $a$. A la acción activa del discente para aprender; $y, b$. El contexto de relación al interior donde se realiza. Estos aspectos sobresalientes constituyen algunas de las características específicas del constructivismo social que, según han definido Bransford, Brown y Coking (2000), constituyen la nueva frontera de la teoría cognoscitiva.

\section{1. ¿Qué es lo que influye en el aprendi- zaje?}

Los autores citados señalan cuatro aspectos que influyen en el aprendizaje:

- Puesto que los discípulos llegan a la clase con un bagaje previo de conocimientos sobre la manera cómo funciona el mundo, si acaso no se valieran de los mismos para aprender, podrían fracasar, ya sea en el entendimiento como en la realización de conexiones con nuevos conceptos e informaciones propuestas.

- Para desarrollar competencias en un sector determinado, los discípulos tienen que: 1 . Poseer una base profunda de cono- 


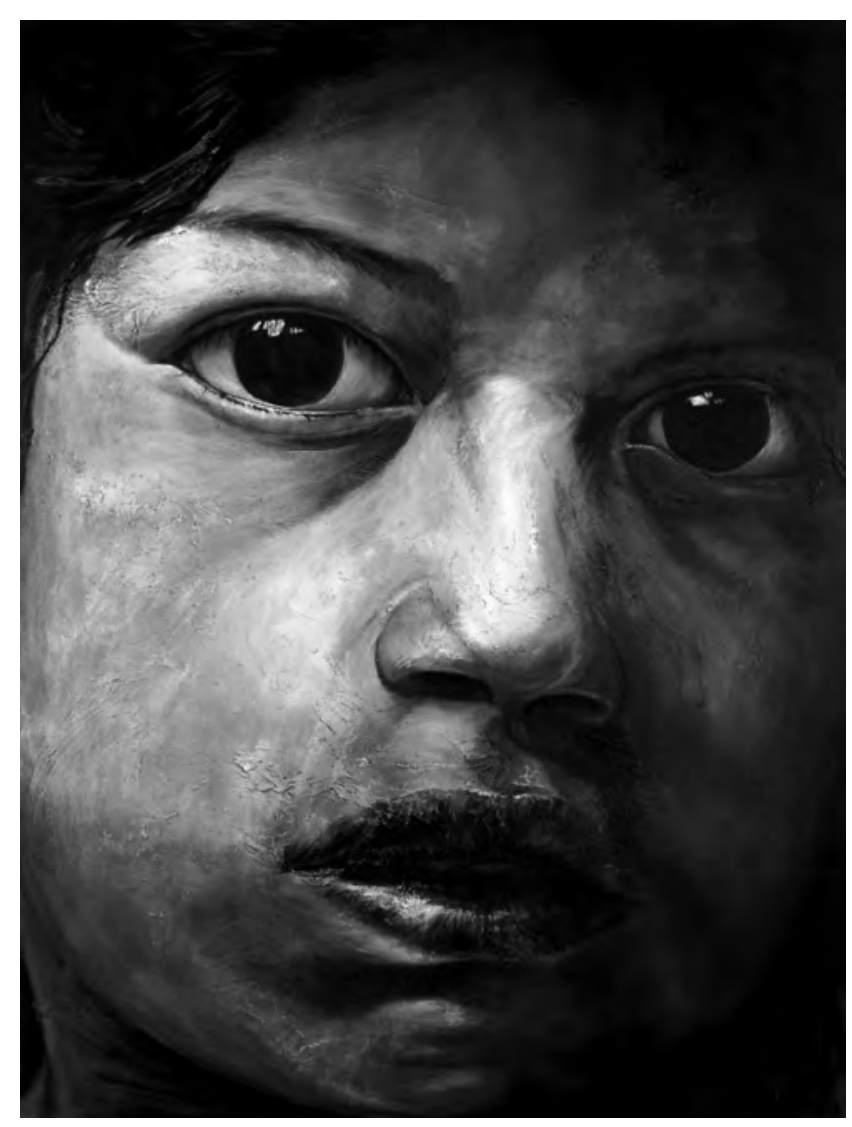

Quilago (32 módulos). Acrílico sobre tela. 2004

cimientos reales; 2. Entender los hechos y las ideas que caracterizan "el pensamiento y la acción" del contexto de qué se trata (disciplinario, profesional, social, etcétera); y, 3. Organizar sus conocimientos de tal manera que pueda facilitar su recuperación y aplicación. Esto supone profundizar la enseñanza de algunos asuntos, suministrando ejemplos de la forma de aplicar el concepto en la realidad.

- Para "aprender a aprender" hace falta aplicar un criterio metacognoscitivo a la instrucción. Las competencias (según el currículo) tendrían que integrarse en el conjunto de los temas de los que se ocupan las disciplinas y ser continuamente auto-evaluadas por los alumnos. De esta forma, ellos mismo pueden aprender a controlar sus aprendizajes, establecer objetivos a alcanzar, monitorear sus adelantos para conseguir dichos objetivos y valorar más la búsqueda de información adicional.

- El contexto influye significativamente en el aprendizaje. Por esta razón, el docente tendría que poner cuidado en el planteamiento de las actividades, de forma que los discípulos constituyan un recurso, los unos para los otros; que el aprendizaje pueda distribuirse en la clase subdividiendo las responsabilidades correspondientes, que se establezca una atmósfera de pensamiento comunitario, en la cual se admiten los conflictos y esté prevista la forma de solucionarlos en cooperación y constructivamente, que la clase se vuelva un contexto de investigación.

\section{2. ¿Qué es lo que se desprende de las in- vestigaciones sobre el aprendizaje?}

La evolución de la investigación sobre las teorías del aprendizaje ha enriquecido mucho el conjunto de conocimientos y experiencias al respecto.

Donovan, Brandsford y Pellegrino (2003), Darling-Hammond y Brandsford (2005), Donovan y Bransford (2005) y Boscolo (2006) sintetizan en otros trabajos algunas respuestas a la pregunta: “¿cómo se aprende, quién es, qué aprende y por qué?”, organizando los resultados alrededor de un punto de vista centrado en el discente. Las características comunes que el aprendizaje supone y que están mencionadas en los trabajos de los autores son las siguientes: 1. La naturaleza constructiva de la cognición; 2. La metacognición; 3. La motivación; 4. El transfer; 5. Las diferencias individuales.

\section{La naturaleza constructiva de la cognición}

Como resultado de las observaciones y argumentaciones científicas de Vygotskij (1962) y Piaget (1975), se puede afirmar que los seres 
humanos -según los autores mencionados-, buscan activamente información y exploran sus expectativas hacia el mundo y cómo el mismo funciona. Ya desde la primera infancia participamos activamente en el intento de interpretar nuestro mundo, valiéndonos de las habilidades, de los conocimientos y de los niveles de desarrollo existentes. Este último, según nos recuerda Vygotskij, se logra inicialmente a través de la acción del aprendizaje, cuya interiorización está facilitada por el apoyo obtenido del contexto (adulto, docente, compañero). Es fundamental la forma en la cual se organiza la actividad para aprender en la clase.

\section{La metacognición}

Cornoldi (1995), Boscolo (1997), Bransford, Brown y Coking (2000), desarrollando las primeras tesis de Flavell (1976) y Brown (1978) demuestran que la conciencia y el control de los procesos cognoscitivos propios constituyen la base para aprender y solucionar problemas. La metacognición es un término que se utiliza para indicar la conciencia del individuo de sus propios procesos cognoscitivos.

En realidad, al interior del gran contenedor de la metacognición que ha desarrollado varias teorías -algunos ejemplos de procesos cognoscitivos de control están constituidos, por ejemplo, por la comprensión de la tarea y la evaluación de las dificultades y de la importancia de lo que se está realizando-, la evaluación de las habilidades y recursos propios, la puesta a punto de las estrategias de las cuales es posible valerse, la decisión y planificación, el monitoreo de la atención y del cumplimiento del plan, la evaluación de los resultados de la acción propia, sacando las consecuencias de todo ello.

\section{La motivación}

Marzano (2003: 144-148), ha correlacionado varios estudios sobre la motivación, demostrando que la misma depende estrictamente del rendimiento y del éxito obtenido en el aprendizaje. Cinco grandes teorías convergen suministrando un cuadro coherente de las consecuencias de la motivación en el aprender, que son las siguientes: 1. Competencia; 2. Autodeterminación; 3. Concepto y percepción de sí mismo; 4. Motivación a conseguir éxito; 5. Atribución (Boscolo, 1997, Marzano, 2003). A éstas Stipek (2003) ha añadido la teoría más moderna de la orientación sociocultural que pone de manifiesto el hecho de que un contexto social adecuado -compañeros, docentes, escuela y padresapoya el sistema motivador, ayudando en particular a persistir enfrentándose con dificultades, y asimismo el que es necesario que cada discente se enfrente efectivamente con un nivel correcto de dificultades, con unos desafíos aptos para envolverlo.

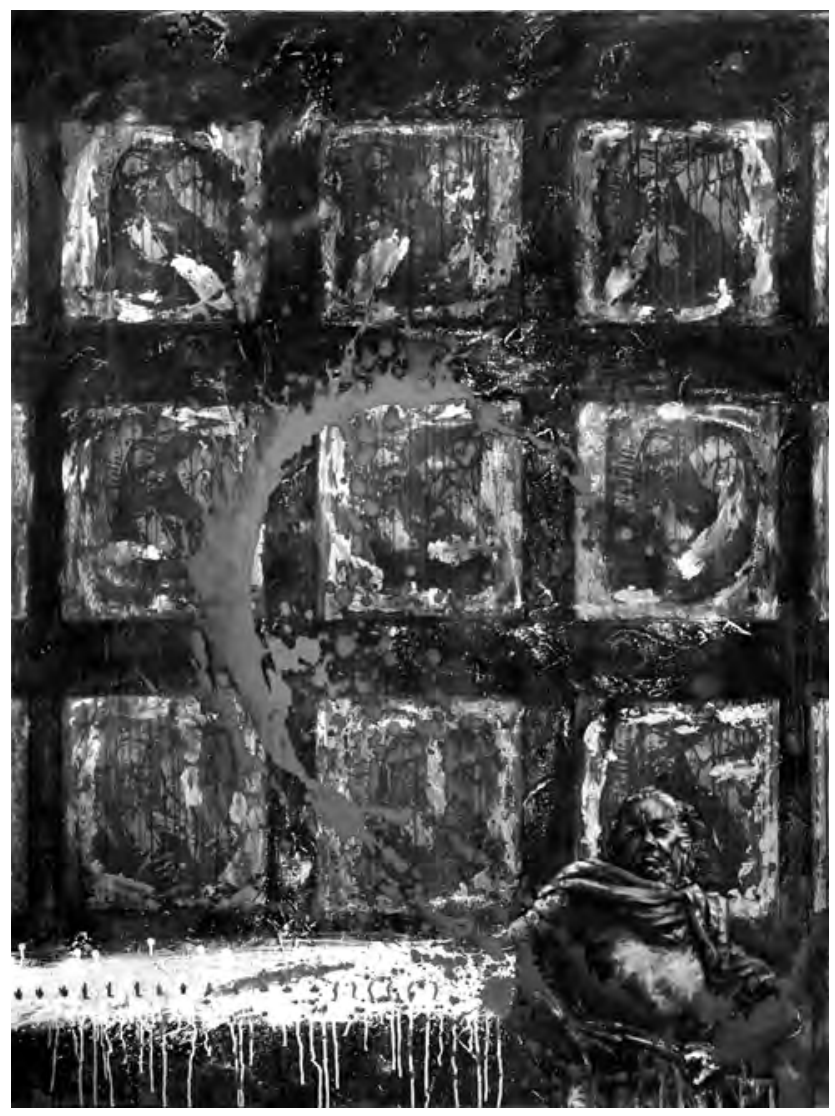

Afirmaciones y negaciones. Acrílico sobre tela. 1996 
Entre los factores que influyen en la motivación -planteamiento compartido por varios autores- observamos, entre otros, la capacidad de controlar las emociones propias, como, por ejemplo, el ansia o la excitación en la autoestimación. Este último concepto, en sus implicaciones sobre el aprendizaje, ha sido investigado en Italia por Franta-Colasanti (1991) y Becciu-Colasanti (2004).

\section{El transfer}

La práctica metacognoscitiva, que permite aprender por sí mismos "cómo se aprende", envuelve un aspecto del pensamiento, el "trasladar", con el significado de aprender las modalidades que nos permiten solucionar unos nuevos problemas con los cuales podríamos enfrentarnos en lo sucesivo (Darling-Hammond, 2005: 61). Según Pellerey (2004: 81), refiriéndose a las investigaciones más modernas sobre el cognitivismo, el transfer no constituye nunca la consecuencia mecánica de un aprendizaje anterior, sino el resultado de una actitud "vigilante" de quien aprende, que le permite valerse de lo que ya sabe sobre un problema parecido a aquel actual, por un proceso de autoregulación. En la práctica, la definición de transfer se vale de las categorías típicas del cognitivismo -analizar, seleccionar, utilizar estrategias, controlar, estar conscientes, organizar- aplicándolas para mejorar las capacidades de aprendizaje.

Al lado de esta idea hay el planteamiento de Bereiter (1995) quien propone el transfer de las situaciones en las cuales ciertas disposiciones del sujeto se han formado: por ejemplo, participar en unas deliberaciones complejas, argumentar ante unos expertos. Este autor propone hacer posible para los discentes recrear las situaciones con las cuales se han enfrentado durante su aprendizaje, actuando en aquellas disposiciones y competencias con varios participantes y condiciones: se pide construir y compartir activamente unos conocimientos.

\section{Las diferencias individuales}

Según afirman Jonassen y Grabowski (1993), los individuos difieren entre sí por una serie de motivos: 1 . Por sus actitudes y preferencias en la elaboración de la información, en la construcción del significado y su aplicación a nuevas situaciones; 2. Por su capacitación para realizar tareas o alcanzar unos resultados diferentes; 3. Por su empleo diferente de competencias, actitudes y preferencias enfrentándose con unas tareas de distinta naturaleza. Estas diferencias influyen en las habilidades de los educandos para alcanzar los resultados de aprendizaje.

\section{- La inteligencia}

Desde el decaimiento del planteamiento psicométrico, no es ventajoso intentar medir las habilidades de las personas por una dimensión única. Las teorías de Gardner (1983) sobre las inteligencias múltiples; de Sternberg (1985) sobre la inteligencia triárquica; de Brown y Campione (1982, 1985), según los cuales hay que concentrarse en las maneras en las cuales los individuos difieren los unos de los otros y no limitarse a definir las habilidades mentales por medio de tests han permitido que tuviese aceptación el principio de la inteligencia no "unívoca", que por consiguiente puede actuar a nivel individual en formas distintas. Se está demostrando además que la inteligencia es educable y que se pueden organizar unos currículos y ambientes de aprendizaje, aptos para potenciar ya sea su desarrollo como el aprendizaje mismo (Perkins 1992, Resnick y Nelson-LeGall, 1998).

Paralelamente, en el estudio de las diferencias individuales influye la investigación sobre los estilos cognoscitivos y de aprendizaje.

\section{- Estilos cognoscitivos}

Plantea Messick (1984: 61), que los estilos cognoscitivos son características cognoscitivas globales que ponen de manifiesto una tendencia individual de funcionamiento cognoscitivo 
(pensar, memorizar, decidir) que reflejan unas constantes en el procesamiento de la información y se desarrollan alrededor de unas orientaciones subyacentes de la personalidad.

Los autores Jonassen y Grabowki (1993: 173-174), plantean que los estilos cognoscitivos juegan un papel en la obtención y organización de la información. Los mismos constituyen unos rasgos estables que los discentes utilizan en su percepción de la información y de los estímulos, mientras que interactúan con el ambiente.

Estas características globales se refieren no sólo en el funcionamiento cognoscitivo, sino también en las actitudes del individuo, en las maneras de ponerse en relación con los demás o de reaccionar a unas situaciones insólitas. Varios autores han redactado varias listas, con unas clasificaciones bipolares que oponen dos orientaciones distintas: visiva-táctil; visiva-verbal; indiferenciadaexacta; serial-holística; analítica-relacional.

\section{- Los estilos de aprendizaje}

En cuanto a los estilos de aprendizaje Jonassen y Grabowski (1993: 233), dicen que son las diferentes tendencias generales las que causan preferencias en el procesamiento de la información. Hay distintas orientaciones respecto a los estilos de aprendizaje con características diferentes. Según el conocido planteamiento de Kolb, el estilo de aprendizaje es un método que una persona prefiere para percibir y elaborar la información. Unos ejemplos de los estilos de este autor son los siguientes: divergente, asimilador, convergente y acomodador.

En esta línea Dunn y Dunn señala que los estilos de aprendizaje son las modalidades preferidas por un discente respecto a la mejor manera de conseguir concentración y el aprender información difícil. La importancia está en las interacciones: $a$. Con el ambiente: algunas bipolaridades son, por ejemplo, silencio-sonido, luz baja-luz fuerte; $b$. Con los demás: aprendizaje individualcolaborativo, presencia de autoridad-ausencia de autoridad, repetición-creatividad; $c$. Con el cuer-

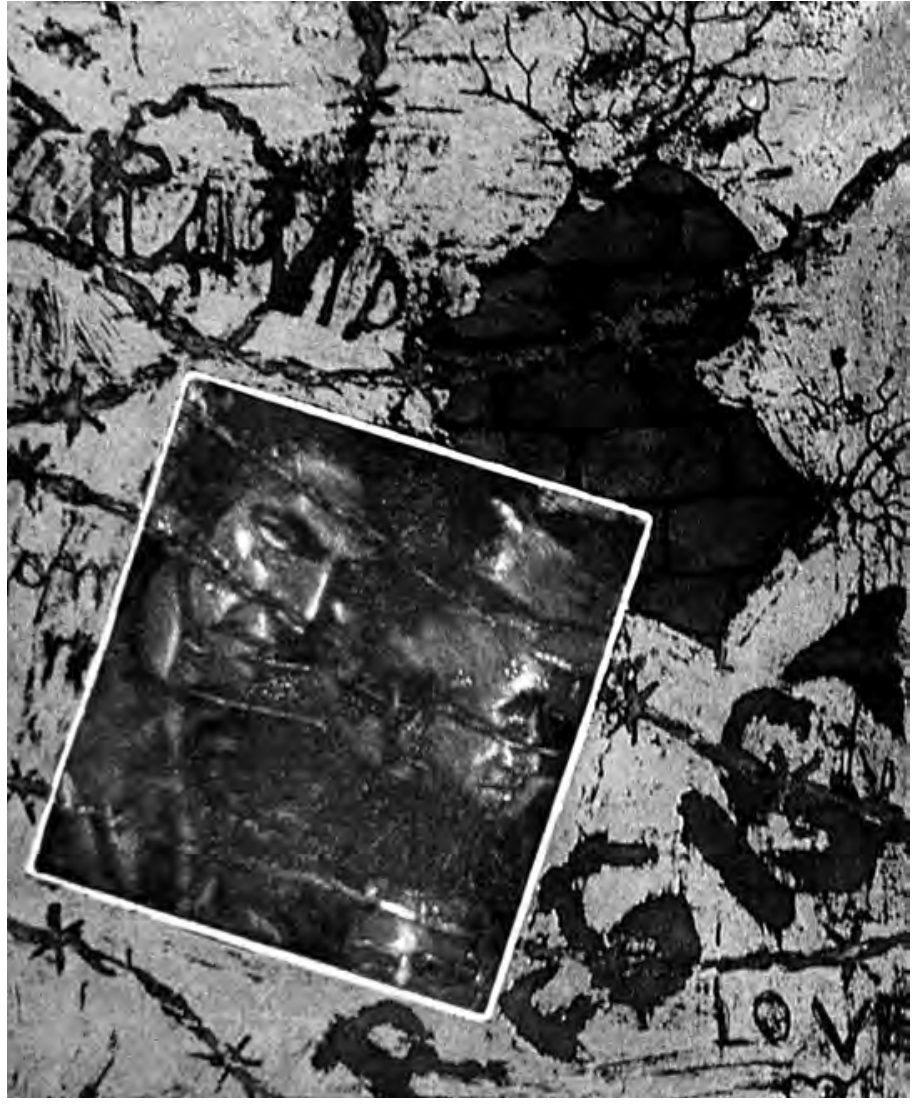

El triunfo de la libertad es el nombre de una calle I. Detalle. Roll up. 1996

po propio: preferencia entre el sistema auditivo visual- táctil - kinésico - movimiento - parado.

Todo lo expuesto arriba confirma el planteamiento de Cornoldi (1991: 45): los estilos de aprendizaje guardan relación estricta con los potenciales individuales de aprendizaje, puesto que constituyen la manera según la cual cada individuo se enfrenta con la realidad y alcanza su conocimiento. Por estas características suyas, los estilos de aprendizaje se adquieren y se modifican por un proceso de desarrollo al interior de un contexto rico en relaciones recíprocas. Es posible, por lo tanto, aprender a incrementar la gama de la cual disponemos, para enfrentarnos con unos contextos y problemas de aprendizaje cada vez nuevos. 
Nos encontramos en una fase de pasaje de los conocimientos sobre el aprendizaje, haciendo hincapié en "cómo" y "qué" es lo que hace falta para aprender. Hay que crear unos "ambientes" en los cuales aplicar las teorías y construir una cultura del aprendizaje -en la clase, en la escuela, entre colegas- promocionando las "artes cognoscitivas" y estableciendo un contexto de vitalidad, de investigación y de entusiasmo para el aprendizaje.

\section{Bibliografía}

- BECCIU, M. Y A. R. Colasanti: La promozione delle capacità personali. Teoria e prassi. Milano: FrancoAngeli. 2004

- BEREITER C.: A dispositional view of transfer. En

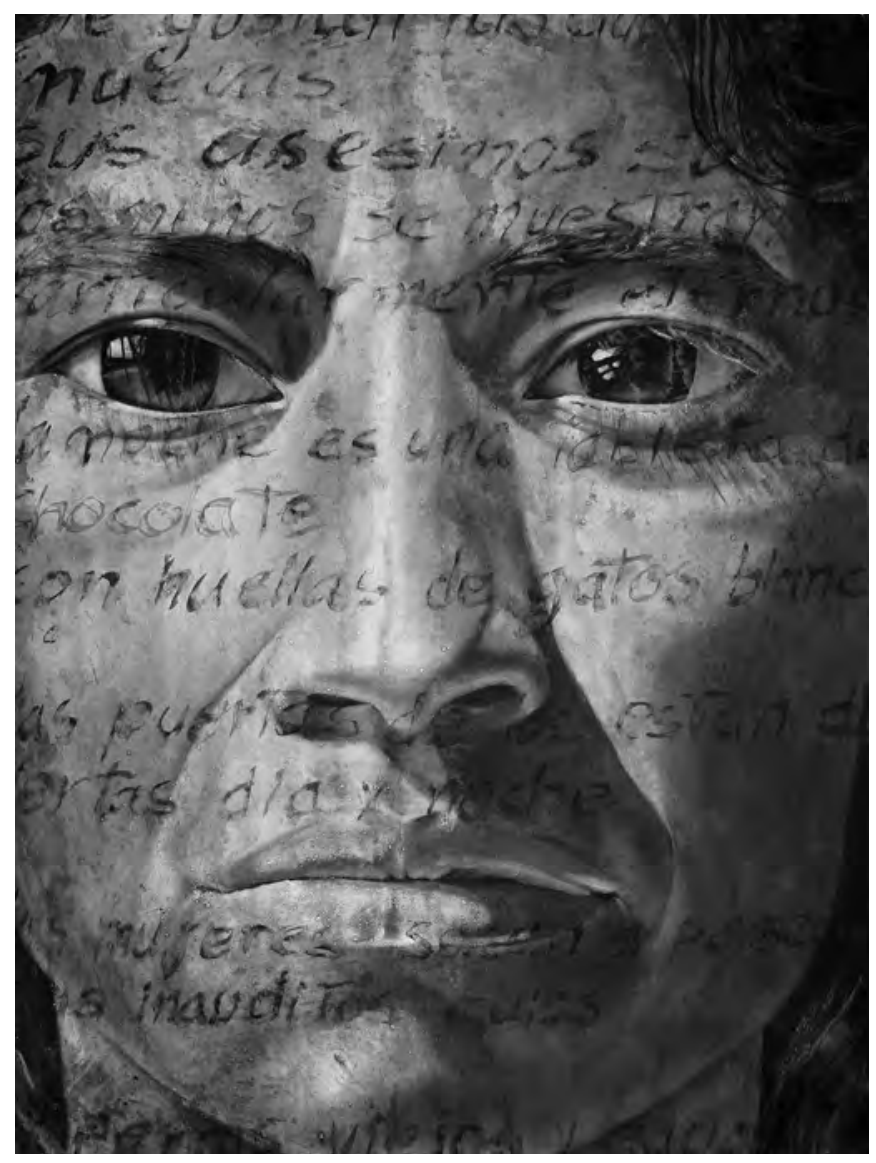

Desarraigo. Acrílico sobre lona. 2008
McKeough - Lupart - Marini (a cura de) Teaching for transfer. Mahwah: Erlbaum. 1995

- BLYTHE, T.: (a cura de) The Teaching for Understanding guide. San Francisco: Jossey-Bass, 1998

- BOSCOLO, P.: Psicologia dell'apprendimento scolastico. Torino: Utet, 1997. nuova edizione 2006

- BOSCOlO, P.: Apprendimento in Cerini G., Spinosi M. (a cura de) Voci della Scuola 2002, Napoli: Tecnodid. 2001. p.11.

- BRANSFORD, J.D., A. Brown, R.Cocking: How people learn. Brain, mind, experience and school. Washington: National Accademy Press. 2000

- BROOK, J. G. y M. Brooks: The case for constructivist classrooms, Alexandria: ASCD. 1999

- BROWN, A.: Knowing when, where, and how remember. A problem of metacognition, in Glaser R. (a cura di) Advances in instructional psychology, vol. I, Hillsdale: Erlbaum. 1978

- BRUNER, J.: La cultura dell'educazione. Milano: Feltrinelli. 1997. (The Culture of Education, Cambridge, Mass.: Harvard University Press. $224+$ xvi pp.)

- CAMPIONE, J.C., A. Brown y N. R. Bryant N.R.: Individual differences I learning and memory. in Sternberg R.J. (a cura de) Human abilities. San Francisco: Freeman. 1985

- CAMPIONE, J.C., A. Brown y R. A. Ferrara: Mental retardation and intelligences, in Sternberg R.J. (a cura de) Handbook of human intelligence. Cambridge: Cambridge University Press. 1982

- Commissione Europea, Competenze chiave per l'apprendimento permanente, Bruxelles: UE. 2006

- CORONOLDI, C.: Stili di insegnamento, stili di apprendimento e handicap. Bergamo: Juvenilia. 1991

- CORNOLDI, C.: Metacognizione e apprendimento. Bologna: Il Mulino, 1995

- DARLING-HAMMOND, L. y J. D. Bransford: Preparing teachers for a chancing world. San Francisco: Jossey-Bass. 2005

- DONOVAN, S. y J. D. Bransford: How student learn. History, mathematics, and science in the classroom. Washington: National Academy Press. 2005

- DONOVAN y Bransford Pellegrino: How People Learn Bridging Research and Practice. Washington: National Research Council. 2004

- EISNER, E.: Preparing for today and tomorrow, Educational Leadership, 61, 4, pp. 6-10. 2003

- ELLERANI, P.: Il cooperative learning. Napoli: Tecnodid. 2003 
- FRABBONI, F.: Società della conoscenza e Scuola. Trento: Erickson, pp. 87-97. 2005

- FLAVELL, J.H.: Metacognitive aspects of problem solving. En Resnick L.B. (a cura de) The nature of intelligence. Hillsdale: Erlbaum. 1976

- FRANTA, H. Y A. R. Colasanti A.R.: L'arte dell'incoraggiamento. Firenze: La Nuova Italia. 1991

- GARDNER H.: Frames of mind. The theory of multiple intelligences. New York: Basic Books, 1983

- JONASSEN, H.J. y B. L. Grabowski: Handbook of individual difference, learning and instruction. Hillsdale: Erlbaum. 1993

- MC TIGHE, J. y G. Wiggins: Understanding by design. Alexandria: ASCD, 1998

- MARZANO, R. J.: What works in schools. Translating research into action. Alexandria: ASCD, 2003

- MESSICK, S.: The nature of cognitive styles, problems and promises in educational practice. Educational Psychologist, 29, pp. 59-74. 1984

- PELLERY, M.: Le competenze individuali e il portfolio. Firenze: La Nuova Italia. 2004

- PERKINS, D.: Schools: From Training Memories to Educating Minds. New York: Free Press. 1992
- PERKINS, D.: The many face of Constructivism. Educational Leadership 57, 3, pp. 6-11. 1999

- PERKINS, D.: Knowledge alive, Educational Leadership 62, 1, pp. 14-18. 2004

- PIAGET, J.: L'equilibrio delle strutture cognitive. Problema centrale dello sviluppo. Torino: Borighieri. 1981 (ed. francese 1975)

- RESNICK, L.B. y Nelson LeGall S.: Socialising intelligences, in Smith L., Dockrell, Tomlinson P., Piaget, Vygotskij and beyond: future issues for developmental psychology and education, London: Routledge, pp. 145-158. 1998

- STERNBERG, H. y I. Q. Beyond: Toward a triarchic theory of intelligence, Cambridge: Cambridge University Press, 1985.

- STIPECK, D.: Engaging Schools: Fostering High School Students' Motivation to Learn, Washington: National Academy Press. 2003

- STONE WISKE, M. (a cura di): Teaching for Understanding. San Francisco: Jossey-Bass, 1998

- VARISCO, B. M.: Costruttivismo socio-culturale. Roma: Carocci. 2002

- VYGOTSKIJ, L.S.: Pensiero e linguaggio. Firenze: Universitaria Barbera. 1962 